\title{
THE RATE OF SPATIAL DECAY OF NONNEGATIVE SOLUTIONS OF NONLINEAR PARABOLIC EQUATIONS AND INEQUALITIES
}

\author{
ALAN V. LAIR \\ (Communicated by Barbara L. Keyfitz)
}

\begin{abstract}
Let $L$ be a uniformly parabolic linear partial differential operator. We show that nonnegative solutions of the differential inequality $L u \leq$ $c(u+|\nabla u|)$ on $\mathbf{R}^{n} \times(0, T)$ for which $u(x, T)=\mathbf{0}\left(\exp \left(-\delta|x|^{2}\right)\right)$ must be identically zero if the constant $\delta$ is sufficiently large. An analogous result is given for nonlinear systems.
\end{abstract}

Let $L$ be the uniformly parabolic linear partial differential operator defined by

$$
L u \equiv \sum_{i, j=1}^{n} \frac{\partial}{\partial x_{j}}\left[a_{i j}(x, t) \frac{\partial u}{\partial x_{i}}\right]-\frac{\partial u}{\partial t} \quad \text { on } D \equiv \mathbf{R}^{n} \times(0, T) .
$$

Landis and Oleinik [6] conjectured that bounded solutions of the equation

$$
L u=g(x) u \text { on } D,
$$

which decrease on a characteristic at the rate $\exp \left(-|x|^{-2-\varepsilon}\right), \varepsilon>0$, must be identically zero. Since then a handful of authors has successfully solved this and related problems. Among these Gusarov [5] showed that solutions of (1) which are $0\left(\exp \left(\gamma|x|^{2}\right)\right)$ satisfying $u(x, T)=\mathbf{0}\left(\exp \left(-\alpha|x|^{2}\right)\right)$ for $\alpha$ sufficiently large must be identically zero. In proving this, the function $g$ and the spatial derivatives of $\left(a_{i j}\right)$ are required to decay to zero as $|x|$ approaches infinity. (He assumes $a_{i j}$ is independent of $t$.) Watson [7] showed that nonnegative solutions of a wider class of linear equations for which $u(x, T)=\mathbf{0}\left(\exp \left(-\alpha|x|^{2}\right)\right)$ must also be trivial. Later these results were extended in two different directions. First, Chabrowski and Watson [2] proved analogous results for nonnegative solutions of weakly coupled linear systems, and second, by specializing to the heat equation, Watson [8] proved slightly stronger results than both [5] and [7].

The purpose of this article is to show that the results for nonnegative solutions of linear equations and systems extend to nonlinear equations, inequalities, and systems. In particular, we prove that the results of Watson [7] and Chabrowski

Received by the editors February 23, 1990 and, in revised form, June 20, 1990.

1980 Mathematics Subject Classification (1985 Revision). Primary 35K55. 
and Watson [2] apply to a much broader class of problems by showing that the only nonnegative solution of

$$
L u \leq c[u+|\nabla u|] \text { on } D(c \geq 0),
$$

$\left(\nabla \equiv\left(\partial / \partial x_{1}, \ldots, \partial / \partial x_{n}\right)\right)$ satisfying

$$
0 \leq u(x, T) \leq C e^{-\delta|x|^{2}}, \quad \delta>\alpha / T>0, C \geq 0, x \in \mathbf{R}^{n},
$$

is the trivial one. The positive constant $\alpha$ comes from (5) below. It should be noticed that inequality (2) applies to $L u=f(x, t, u, \nabla u)$, where $f(x, t, u, \mathbf{p})$ need only be bounded above by a multiple of $(u+|\mathbf{p}|)$ for nonnegative $u$ and for all $\mathbf{p} \in \mathbf{R}^{n}$. Since the equations in $[2,7]$ are linear and are required to have bounded coefficients, our results contain those as a special case. Furthermore, the lower bound $\alpha / T$ on the size of $\delta$ matches precisely those of $[2,7]$.

Let $C^{*}(D)$ be the set of functions $u$ which are continuous on $\bar{D}$ (the closure of $D$ ) and such that $u_{t}, u_{x_{i} x_{j}}, 1 \leq i, j \leq n$, are continuous on $D$. We assume the matrix $\left(a_{i j}\right)$ is symmetric on $\bar{D}$ and there exists a constant $\lambda>0$ such that

$$
a_{i j}(x, t) \xi_{i} \xi_{j} \geq \lambda|\xi|^{2}, \quad \xi \in \mathbf{R}^{n},(x, t) \in D .
$$

(The summation convention is used on repeated indices.) Furthermore, we assume the functions $a_{i j}$ and $\partial a_{i j} / \partial x_{m}, 1 \leq i, j, m \leq n$, are locally Höldercontinuous and bounded on $D$. We note that under these conditions, the equation $L u=0$ has a fundamental solution $\Gamma(x, t ; \xi, \tau)$ (see [4]) with the following property (see [1]): there exist positive constants $\alpha$ and $K$, depending only on $\lambda, n$, and the bounds for $a_{i j}$ and $\left(a_{i j}\right)_{x_{m}}$, such that

$$
K(4 \pi(t-\tau))^{-n / 2} \exp \left(-\alpha|x-\xi|^{2} /(t-\tau)\right) \leq \Gamma(x, t ; \xi, \tau)
$$

for $(x, t),(\xi, \tau) \in \mathbf{R}^{n} \times(0, T]$, with $\tau<t$.

Lemma 1. Suppose $v \in C^{*}(D)$ is a nonnegative function satisfying

$$
\int_{\mathbf{R}^{n}} \Gamma(x, t ; \xi, \tau) v(\xi, \tau) d \xi \leq v(x, t), \quad 0 \leq \tau<t \leq T,
$$

and

$$
v(x, T) \leq C e^{-\zeta|x|^{2}} \text { for some } \zeta>\alpha / T \text { and all } x \in \mathbf{R}^{n} .
$$

Then $v(x, T)=0$ for all $x \in \mathbf{R}^{n}$.

Proof. The proof of this is very similar to the proofs of [7, Theorems 1 and 2], and hence we merely give an outline here. Let $M(r)=\max \{v(x, T):\|x\|=r\}$ and proceed as in [7] to show that inequalities (5), (6), and (7) are incompatible with the existence of $x_{0} \in \mathbf{R}^{n}$ for which $v\left(x_{0}, T\right)>0$. (Our $\alpha$ corresponds to $\alpha^{2} / 4$ in [7].) Thus $v(x, T)$ must be identically zero. 
Lemma 2. Suppose $v \in C^{*}(D)$ is a nonnegative solution of $L v \leq 0$ on $D$. Then

$$
\int_{\mathbf{R}^{n}} \Gamma(x, t ; \xi, \tau) v(\xi, \tau) d \xi \leq v(x, t), \quad 0 \leq \tau<t \leq T, x \in \mathbf{R}^{n} .
$$

Proof. Define

$v_{R}(x, t)=v(x, t)-\int_{|\xi| \leq R} \Gamma(x, t ; \xi, \tau) v(\xi, \tau) d \xi, \quad 0 \leq \tau<t \leq T, x \in \mathbf{R}^{n}$.

Using an argument similar to that of [3, p. 507], we get

$$
\liminf _{(x, t) \rightarrow\left(x_{0}, \tau^{+}\right)} v_{R}(x, t) \geq 0, \quad x_{0} \in \mathbf{R}^{n}, \tau \geq 0 .
$$

Considering the hypothesis and the properties of $\Gamma$, we have $L v_{R}=L v \leq 0$ on $\mathbf{R}^{n} \times(0, T)$. Thus we get $v_{R}(x, t) \geq 0$ for $(x, t) \in \mathbf{R}^{n} \times(\tau, T]$ and hence

$$
\int_{|\xi| \leq R} \Gamma(x, t ; \xi, \tau) v(\xi, \tau) d \xi \leq v(x, t), \quad 0 \leq \tau<t \leq T, x \in \mathbf{R}^{n} .
$$

Noting that the left-hand side of this expression is monotonic in $R$, we get its convergence as $R \rightarrow \infty$, and thus the lemma is proved.

Theorem 1. If $u$ is a nonnegative $C^{*}(D)$ solution of (2) and (3), then $u \equiv 0$ on $\bar{D}$.

Proof. Define the set $S=\left\{t \in(0, T]: u(x, t)=0\right.$ for some $\left.x \in \mathbf{R}^{n}\right\}$. Note that if $\tilde{t} \in S$, then $u \equiv 0$ on $\mathbf{R}^{n} \times[0, \tilde{t}]$, for if we let $w(x, t)=e^{c t} u(x, t)$ and rewrite (2) as $L w-c(\nabla w /|\nabla w|) \cdot \nabla w \leq 0$, we may apply the strong maximum principle to get $w \equiv 0$ (and hence $u \equiv 0$ ) on $\mathbf{R}^{n} \times[0, \tilde{t}]$. Therefore, to complete the proof, we let $t_{0}=\sup (S)$ and prove $t_{0}=T$. Thus suppose $t_{0}<T$ and hence $u(x, t)>0$ on $\mathbf{R}^{n} \times\left(t_{0}, T\right]$. Let $v=e^{\sigma t} u^{\nu}$, where $\sigma$ is a large positive constant to be chosen momentarily and $\nu$ is a constant in the open interval $(0,1)$ chosen near unity, so that $\nu \delta>\alpha / T$. Applying the operator $L$ to $v$ and using (2) and (4), we obtain

$$
\begin{aligned}
e^{-\sigma t} L v & \leq \nu u^{\nu-1} L u+\nu(\nu-1) u^{\nu-2} a_{i j} u_{x_{i}} u_{x_{j}}-\sigma u^{\nu} \\
& \leq(c \nu-\sigma) u^{\nu}+c \nu u^{\nu-1}|\nabla u|+\lambda \nu(\nu-1) u^{\nu-2}|\nabla u|^{2} .
\end{aligned}
$$

Now apply the inequality $u^{\nu-1}|\nabla u| \leq(4 \varepsilon)^{-1} u^{\nu}+\varepsilon u^{\nu-2}|\nabla u|^{2}, \varepsilon>0$, to get

$$
e^{-\sigma t} L v \leq(c \nu-\sigma+c \nu / 4 \varepsilon) u^{\nu}+(\lambda \nu(\nu-1)+\varepsilon c \nu) u^{\nu-2}|\nabla u|^{2} \leq 0,
$$

where the last inequality holds for $\varepsilon>0$ sufficiently small and $\sigma$ sufficiently large. With $\varepsilon$ and $\sigma$ so chosen, we get $L v \leq 0$ on $\mathbf{R}^{n} \times\left(t_{0}, T\right)$ and since $v$ is positive, Lemma 2 yields

$$
\int_{\mathbf{R}^{n}} \Gamma(x, t ; \xi, \tau) v(\xi, \tau) d \xi \leq v(x, t), \quad 0 \leq t_{0}<\tau<t \leq T, x \in \mathbf{R}^{n} .
$$

Since $u$ satisfies (3), we get $0 \leq v(x, T) \leq C_{T} e^{-\delta \nu|x|^{2}}$, where $\delta \nu>\alpha / T$ and $C_{T}=C^{\nu} e^{\sigma T} \geq 0$. Hence Lemma 1 yields $v(x, T)=0$ for all $x \in \mathbf{R}^{n}$, 
implying $T \in S$. Thus our assumption that $t_{0}<T$ leads to the conclusion that $T \in S$ which, from the definition of $t_{0}$, is impossible. Therefore $t_{0}=T$ and the proof is complete.

We prove an analogous result for nonlinear systems which includes, as a special case, a similar result of Chabrowski and Watson [2, Theorem 6]. In particular, we consider systems of inequalities

$$
\begin{aligned}
& L^{k} u^{k}+f^{k}\left(x, t, u^{1}, \ldots, u^{N}, \nabla u^{1}, \ldots, \nabla u^{N}\right) \\
& \quad \leq c\left[u^{k}+\left|\nabla u^{k}\right|\right], \quad(x, t) \in D, 1 \leq k \leq N,
\end{aligned}
$$

where the operators $L^{k}$ are defined by

$$
L^{k} u^{k} \equiv\left(a_{i j}^{k}(x, t) u_{x_{i}}^{k}\right)_{x_{j}}-u_{t}^{k} .
$$

As earlier, we assume the coefficients $\left(a_{i j}^{k}\right)$ are sufficiently nice that the fundamental solution of the equation $L^{k} v=0$ exists and satisfies (5) for each value of $k$. Furthermore, we assume there exists a nonnegative constant $\kappa$ such that

$$
f^{k}\left(x, t, u^{1}, \ldots, u^{N}, \mathbf{p}^{1}, \ldots, \mathbf{p}^{N}\right) \geq-\kappa\left(u^{k}+\left|\mathbf{p}^{k}\right|\right)
$$

for all nonnegative $u^{1}, \ldots, u^{N}$, all $\mathbf{p}^{1}, \ldots, \mathbf{p}^{N}$ in $R^{n}$, and $(x, t) \in D$. Note that if $f^{k}$ is linear in the variables $u^{1}, \ldots, u^{N}, \mathbf{p}^{1}, \ldots, \mathbf{p}^{N}$, then (9) corresponds to the requirement in [2, Theorem 6] that the coefficients on $u^{1}, \ldots$, $u^{k-1}, u^{k+1}, \ldots, u^{N}$ be nonnegative and the coefficients on $u^{k}$ and $\mathbf{p}^{k}$ be bounded.

Theorem 2. Suppose the functions $f^{k}$ satisfy (9) and $\left\{u^{1}, \ldots, u^{N}\right\}$ is a nonnegative $C^{*}(D)$ solution of $(8)$ satisfying

$$
0 \leq u^{k}(x, T) \leq C e^{-\delta|x|^{2}}, \quad \delta>\alpha / T, C \geq 0,1 \leq k \leq N, x \in \mathbf{R}^{n} .
$$

Then $u^{1}=\cdots=u^{N}=0$ on $\bar{D}$.

Proof. Considering (8) and (9), we get that $u^{k}$ is a nonnegative solution of

$$
L^{k} u^{k} \leq \hat{c}\left[u^{k}+\left|\nabla u^{k}\right|\right] \text { on } D, 1 \leq k \leq N,
$$

where $\hat{c}=c+\kappa$ and hence, by Theorem 1 , we get $u^{k}=0$. This completes the proof.

\section{REFERENCES}

1. D. G. Aronson, Bounds for the fundamental solution of a parabolic equation, Bull. Amer. Math. Soc. 73 (1967), 890-896.

2. J. Chabrowski and N. A. Watson, Properties of solutions of weakly coupled parabolic systems, J. London Math. Soc. (3) 23 (1981), 475-495. 
3. A. Friedman, On the uniqueness of the Cauchy problem for parabolic equations, Amer. J. Math. 81 (1959), 503-511.

4. _. Partial differential equations of a parabolic type, Prentice-Hall, Englewood Cliffs, NJ, 1964.

5. A. L. Gusarov, On a sharp Liouville theorem for solutions of a parabolic equation on a characteristic, Math. USSR-Sb. 26 (1975), 349-364.

6. E. M. Landis and O. A. Oleinik, Generalized analyticity and some properties of solutions of elliptic and parabolic equations, Russian Math. Surveys 29 (1974), 195-212.

7. N. A. Watson, The rate of spatial decay of non-negative solutions of linear parabolic equations, Arch. Rational Mech. Anal. 68 (1978), 121-124.

8. __ Parabolic equations on an infinite strip, Marcel Dekker, New York, 1989.

Department of Mathematics and Computer Science, Air Force Institute of Technology, Wright-PATterson AIR Force Base, OHIo 45433 